\title{
Phonetic Analysis of a Computational Model for Vocabulary Acquisition from Auditory Inputs
}

\author{
Hugo Van hamme \\ Department ESAT \\ Katholieke Universiteit Leuven, Belgium \\ Email: hugo.vanhamme@esat.kuleuven.be
}

\begin{abstract}
An overview of the NMF (non-negative matrix factorization) approach for word acquisition from auditory inputs is given. The method can link acoustic realizations of spoken words with information observed in other modalities. The method is applied to the acquisition of a small set of keywords embedded in carrier sentences. A phonetic description is linked to the learned representations which are otherwise difficult to interpret. Through this link, the phonetic similarity between the learned acoustic representations and lexical items is displayed and interpreted.
\end{abstract}

Index Terms-non-negative matrix factorization, pattern discovery, word acquisition.

\section{INTRODUCTION}

Billions of babies around the globe have succeeded to acquire a vocabulary of their native language. This is a process with a surprisingly small amount of direct supervision. Most words they know are not explained to them, but are learned from their significance in the world they live in. The task is even more complicated if we observe that most of the utterances we speak to our babies or children contain more than one word [1] and many words are never heard in isolation. Yet, they succeed finally in segmenting spoken utterances into words.

Modeling child language acquisition (CLA) is not the only motivation for this work. In human-machine interaction, there are benefits for the acquisition of a vocabulary by interacting with the machine, a special case of Socially Guided Machine Learning (SGML) [2]. Such a "on-the-job-learning" approach deviates from the predominant design paradigm for vocal interfaces in which a developer decides on the vocabulary and grammar that the end-user will need to adopt. The user will then have to refer to concepts, objects and actions in terms that he/she might not naturally choose. In contrast, our interest lies in technology that can acquire a vocabulary in any language without the need to initialize it with prior knowledge about the user's language. In particular, such technology could be useful for people with a motor impairment making traditional input devices such as keyboards, mice or push-buttons difficult to use. However, it is not unlikely that the user's speech is also affected, resulting in an unusual phone inventory. Like in CLA, it is thus too strong an assumption to start from a phoneme or even phone inventory.
Both in modeling CLA and in SGML, a first task seems to be finding the words in running speech. One of the difficulties that need to be overcome is that the transcription of speech into symbols such as phones or even letters is not free of error. In the DP-N-gram-approach [3], partial matches between two strings of phonetic transcriptions generated with an HMMbased phone transcriber are found using a dynamic programming algorithm such that the matched transcriptions should be similar but not identical. Subsequently, an agglomerative clustering algorithm groups the similar phone strings into clusters that are then used for topic spotting. In [4], dynamic time warping at the acoustic level comparing MFCC vectors directly is used instead of matching through dynamic programming at a symbolic level. The units that are most similar in this fault-tolerant match are retained as acoustic patterns. In the multigram approach of [5], a symbolically labeled input stream is entirely explained as a sequence of a finite vocabulary of multigram models of variable length. Each of the vocabulary entries emits patterns of variable length according to a statistical model. Estimation and detection with multigrams are scrutinized and an extension to acoustic (non-symbolic) input using "temporal decomposition" is proposed.

Discovering word-sized acoustic patterns that recur in speech is however not sufficient: they also need to be linked to events in other modalities such as vision, touch, taste and smell, for CLA or pressing buttons and the like in SGML. In [6], a cognitively more plausible model of early lexical acquisition is proposed. A short-term recurrence filter finds similar patterns in automatically generated phonetic representations. The model subsequently links the acoustic pattern discovery with word-to-meaning acquisition by using multisensory input.

The core of the learning algorithm that is proposed in this work is non-negative matrix factorization (NMF) [7]. By imposing non-negativity constraints, NMF allows only additive (not subtractive) combinations of parts, and thereby it is distinguished from other matrix factorization techniques such as principal component analysis or singular value decomposition. The proposed method differs in several respects from the work in [6]: it does not require to be able to segment speech into phonetic event and it uses a different learning paradigm. 


\section{LEARNING WITH NMF}

Learning with NMF can be achieved in a general setting by considering a collection of scenes $\psi_{t}(1 \leq t \leq T)$, each composed of an unordered collection of objects $\omega_{r}(1 \leq r \leq R)$, where $R \ll T$. The $t$-th scene is mapped onto some $N$ dimensional vector space $\mathbf{v}_{t}=f\left(\psi_{t}\right)$, the $N$ entries of which are referred to as features. The image of the objects under $f$ are denoted by $\mathbf{w}_{r}=f\left(\omega_{r}\right)$. The mapping $f$ (see section III) is chosen such that:

$$
\mathbf{v}_{t} \approx \sum_{r=1}^{R} h_{r t} \mathbf{w}_{r}
$$

where weight $h_{r t}$ quantifies how strongly object $r$ is present in scene $t$. With $\mathbf{V}=\left[\begin{array}{lll}\mathbf{v}_{1} & \ldots & \mathbf{v}_{T}\end{array}\right]$ and similar for $\mathbf{W}$ and $\mathbf{H}$ :

$$
\mathbf{V} \approx \mathbf{W} \mathbf{H}
$$

where all matrix entries are non-negative. Finding $\mathbf{W}$ and $\mathbf{H}$ from given data $\mathbf{V}$ in (2) is referred to as a Non-negative Matrix Factorization (NMF) problem, which is solved by minimizing the generalized Kullback-Leibler divergence (KLD) between $\mathbf{V}$ and its model $\mathbf{W} \mathbf{H}$ through iteration [7]. Given a collection of $T$ scenes, it allows to learn the vector space description $\mathbf{W}$ of the $R$ objects without ever having to observe the object alone in a scene. The conditions under which the decomposition is unique, within a trivial permutation and scaling of the columns of $\mathbf{W}$, are given in [9].

\section{A. Learning from multimodal data}

As outlined in the introduction, the objects in the CLA and SGML applications are assumed to be observable in multiple modalities. Formally, this means that the mapping $f$ generates features that relate to more than one modality. In word acquisition (either CLA or SGML), the auditory observation of a scene is an utterance and the objects are observed as words. The NMF learning framework remains valid in the case of multimodal features, as long as the linear relation (1) holds with common weights $h_{r t}$. The object representations W will also contain rows that relate to the features from different modalities, so they become cross-modal representations. Notice that common weights across modalities are debatable, since not all objects in the scene may be observable in all modalities. For CLA, one may talk about an object that is not visible in the scene, or conversely, auditory input will in general not deal with all visible objects. Hence, a model in which weights of an object in different modalities are drawn from a distribution conditioned on a latent variable may be more appropriate [8]. While this is a topic of research in CLA, the assumption is reasonable in SGML.

A special modality is the ground truth, which is represented in the matrix $\mathbf{G}$ with $\mathbf{G}_{i t}$ equal to the number of times object $i$ occurs in scene $t$. Because this scene-level information stream is ideal, one can refer to this case as supervised learning. Likewise, with features from different modalities, one modality acts as (noisy) supervisory information to the other.

\section{B. Feature reconstruction}

After $\mathbf{W}$ has been learned on training data, it can be used in testing, i.e. only $\mathbf{H}$ is estimated on new data $\mathbf{V}$ while $\mathbf{W}$ is fixed. If some features are unobservable, this is tantamount to removing some rows of $\mathbf{V}$. Without loss of generality, we can partition the NMF problem as:

$$
\left[\begin{array}{c}
\mathbf{V}_{k} \\
\mathbf{V}_{u}
\end{array}\right] \approx\left[\begin{array}{l}
\mathbf{W}_{k} \\
\mathbf{W}_{u}
\end{array}\right] \mathbf{H}
$$

where subscript $k$ and $u$ denote known and unknown information respectively. If $\mathbf{V}_{u}$ and $\mathbf{W}_{u}$ are removed, the NMF problem can still be solved for $\mathbf{H}$, without a strong impact on the solution. This is firstly motivated by the fact that the equations (2) are overdetermined and are assumed to remain so after removing rows. Secondly, the non-negativity constraints typically lead to a sparse $\mathbf{H}$ that is not strongly affected by removal of the some of the equations. Subsequently, $\mathbf{V}_{u}$ is estimated as $\mathbf{W}_{u} \mathbf{H}$. In case the unobservable rows all belong to one (or more) modality, this allows the reconstruction of the features of that modality from observations in another modality.

For the special case where the objects are observed in the auditory modality $\left(\mathbf{V}_{\text {aud }}\right)$, the supervised training problem becomes:

$$
\left[\begin{array}{c}
\mathbf{G} \\
\mathbf{V}_{\text {aud }}
\end{array}\right] \approx\left[\begin{array}{c}
\mathbf{W}_{g} \\
\mathbf{W}_{\text {aud }}
\end{array}\right] \mathbf{H}
$$

where $\mathbf{G}$ denotes the ground truth information as before and $\mathbf{W}_{g}$ and $\mathbf{W}_{\text {aud }}$ are stored for testing. During the testing phase, the ground truth is missing and can be reconstructed as explained above, i.e. activations are computed as $\mathbf{A}=\mathbf{W}_{g} \mathbf{H}$, where $\mathbf{H}$ is found by solving the NMF problem $\mathbf{V}_{\text {aud }} \approx \mathbf{W}_{\text {aud }} \mathbf{H}$ for $\mathbf{H}$. If $\mathbf{A}_{i t}$ exceeds a threshold, word $i$ is detected in utterance $t$, i.e. speech recognition was performed.

\section{Choice OF FeAtures}

A histogram of local features observed in the scene is an example of a mapping $f$ that satisfies the linearity property (1). This mapping is motivated by a model in which neurons fire upon recognizing a local feature and neurons upstream are activated by sufficient neural spike counts. Since the features are measured locally, they are hardly affected by their neighbors, so measuring their occurrence frequency over a complete scene is additive. In vision, local features describe the image structure around an interest point, e.g. by SIFT features [10].

\section{A. Sound transition statistics}

For speech, it may not be as obvious what the mapping $f$ should be. Psycholinguistic experiments have shown that one of the cues that babies exploit for finding words in running speech are sound transition statistics [11], i.e. the statistical relationships between sounds differs across words and within words. Since it is inappropriate in CLA and SGML to assume a phonemic sound inventory before words have been acquired, 


\begin{tabular}{|l|l|l|}
\hline auto & Car & 696 \\
\hline bad & Bath & 696 \\
\hline boek & Book & 709 \\
\hline damian & proper name & 176 \\
\hline flesje & Bottle & 708 \\
\hline isabel & proper name & 178 \\
\hline luier & Diaper & 708 \\
\hline mama & mammy & 697 \\
\hline mirjam & proper name & 171 \\
\hline otto & proper name & 175 \\
\hline papa & Daddy & 682 \\
\hline schoen & Shoe & 693 \\
\hline Telefoon & telephone & 712 \\
\hline
\end{tabular}

Table 1: number of occurrences in the training set (right column) of each of the keywords (left column) and their translation (middle column).

the current method is based on observing spectral change statistics as follows. The human ear is modeled with a MELscale filter bank whose log-energy outputs are sampled every 10 milliseconds. This is known to be a coarse, yet workable approximation. As is common in speech recognition technology, the first and second order time derivatives are also computed resulting in three data streams called static (S), velocity $(\mathrm{V})$ and acceleration (A). The input audio will be characterized by its similarity to prototypes in each of these streams. The spectral similarity metric is Euclidean distance after applying the cepstral transform to each of the three streams. Therefore, the observed spectral vectors of each stream $s\left(s=\mathrm{S}, \mathrm{V}\right.$ or A) are clustered into $N_{s}$ centroids using the K-means algorithm. The posterior probabilities $P_{i, s, n}$ of all centroids $n$ now characterize any frame of audio data at frame (time) $i$ in terms of its similarity to each of the centroids. Motivated by the Euclidean distance metric used in clustering, the posteriors are based on a Gaussian model with spherical covariance. The posterior probabilities satisfy:

$$
\sum_{n=1}^{N_{s}} P_{i, s, n}=1 \text { at frame } i \text { and for } s=\mathrm{S}, \mathrm{V} \text { or A }
$$

A special case is obtained in a vector quantization (or "winner takes all") setting, where all posteriors are zero except for the centroid which is closest to the observation, which is assigned the value 1 .

The next step is to construct sound transition features of the scene (utterance) by comparing the centroid posterior probabilities $P_{i, s, n}$ at different times $i$ and $i+\tau$ for some chosen lag $\tau$. Hence, the lag- $\tau$ co-occurrence statistics are formed for each stream $s$ :

$$
\left[\mathbf{C}_{s, t}^{\tau}\right]_{m n}=\sum_{i=1}^{I_{t}-\tau} P_{i, s, m} P_{i+\tau, s, n} \text { with } m, n=1 \ldots N_{s}
$$

where $I_{t}$ is the length (in frames) of the $t$-th utterance. Notice that this co-occurrence is directed: $\left[\mathbf{C}_{s, t}{ }^{\tau}\right]_{n m} \neq\left[\mathbf{C}_{s, t}{ }^{\tau}\right]_{m n}$

Let $\operatorname{vec}(\mathbf{C})$ denote the operator that stacks all columns of $\mathbf{C}$ in one column vector. The data matrix of a set of $T$ utterances

\begin{tabular}{|l|l|r|}
\hline daar is $<$ article $><$ key $>$ & there is $<$ article $><$ key $>$ & 709 \\
\hline (dag $\mid$ hallo|hee $\mid$ hoi $)<$ key $>$ & hello $<$ key $>$ & 288 \\
\hline dat is $<$ article $><$ key $>$ & that is $<$ article $><$ key $>$ & 1103 \\
\hline en daar komt $<$ key $>$ & and there comes $<$ key $>$ & 138 \\
\hline en hier is $<$ article $><$ key $>$ & and here is $<$ article $><$ key $>$ & 697 \\
\hline kijk $<$ article $><$ key $>$ & look $<$ article $><$ key $>$ & 629 \\
\hline pak je $<$ article $><$ key $>$ ? & do you take $<$ article $><$ key $>$ ? & 476 \\
\hline $\begin{array}{l}\text { waar is }<\text { article }><\text { key }> \\
\text { nou? }\end{array}$ & where is $<$ article $><$ key $>$ ? & 712 \\
\hline wat een leuk(e) $<$ key $>$ & what a nice $<$ key $>$ & 413 \\
\hline wijs $<$ article $><$ key $>$ ann & point at $<$ article $><$ key $>$ & 631 \\
\hline zie je $<$ article $><$ key $>$ ? & Do you see $<$ article $><$ key $>$ ? & 694 \\
\hline
\end{tabular}

Table 2: number of occurrences in the training set (right column) of each carrier phrase (left column) and its English translation (middle column).

is now formed by

$$
\mathbf{V}^{\tau}=\left[\begin{array}{llr}
\operatorname{vec}\left(\mathbf{C}_{S, 1}^{\tau}\right) & \operatorname{vec}\left(\mathbf{C}_{S, t}^{\tau}\right) & \operatorname{vec}\left(\mathbf{C}_{S, T}^{\tau}\right) \\
\operatorname{vec}\left(\mathbf{C}_{V, 1}^{\tau}\right) \cdots & \operatorname{vec}\left(\mathbf{C}_{V, t}^{\tau}\right) \cdots & \operatorname{vec}\left(\mathbf{C}_{V, T}^{\tau}\right) \\
\operatorname{vec}\left(\mathbf{C}_{A, 1}^{\tau}\right) & \operatorname{vec}\left(\mathbf{C}_{A, t}^{\tau}\right) & \operatorname{vec}\left(\mathbf{C}_{A, T}^{\tau}\right)
\end{array}\right]
$$

Notice that all entries in the $\left(N_{S}^{2}+N_{V}^{2}+N_{A}^{2}\right) \times T$ matrix $\mathbf{V}^{\tau}$. In case vector quantization is used, $\mathbf{V}^{\tau}$ contains the centroid cooccurrence counts (histogram) and tends to be sparse. Finally, stacking the information for $Q$ different lags yields the histogram of acoustic co-occurrence (HAC):

$$
\mathbf{V}_{\text {aud }}=\left[\begin{array}{c}
\mathbf{V}^{\tau_{1}} \\
\mathbf{V}^{\tau_{2}} \\
\vdots \\
\mathbf{V}^{\tau_{Q}}
\end{array}\right]
$$

\section{EXPERIMENTS}

\section{A. The Dutch ACORNS database}

Two male and two female adult speakers each utter 1000 sentences containing a single keyword in an infant-directed (IDS) and adult-directed (ADS) speaking mode. Of these 7999 utterances, 1000 are randomly drawn for testing, the rest is used for training. The keywords, their translation in English and the number of occurrences in the training set are given in Table 1. There is one and only one keyword per utterance in this experiment, but as shown in [13], this is not a requirement of the method.

Each of the keywords is embedded in a carrier sentence. The different carrier sentences are listed in Table 2. Here, $<$ article> is either empty (for proper names), "de" (definite article for male or female nouns), "het" (neutral nouns) or "een" (indefinite article for all genders), while $<\mathrm{key}>$ is one of the keywords form Table 1. The keywords do not occur with an even distribution over in all carriers. The total number of different words (keyword and carrier) is 37 . 


\section{B. Training and recognition}

In this experiment, a codebook for static, velocity and acceleration features of respectively $N_{S}=150, N_{V}=150$ and $N_{A}=100$ entries is used with the vector quantization approach described in section III. The codebook size is a compromise between quantization error and the size of the matrices to be handled. Training is then achieved by performing NMF with $T=6999$, a common dimension of $\mathbf{W}$ and $\mathbf{H}$ of 25 and a number of rows $N$ that varies depending on $Q$, i.e. the number of different lag values $\tau$ that is considered. Up to three lags are used as listed in the first three columns of Table 3. Rows of $\mathbf{V}$ in equation (3) that are zero in the training are removed and the resulting number of rows $N$ of $\mathbf{V}$ is given in column 4 of Table 3. Subsequently, the activations of the 13 keywords is computed as described in section II.B, the keyword with maximal activation is selected and the resulting error rate on 1000 test utterances is listed in the rightmost column of Table 3.

Most tags are correctly recognized despite the different speaking styles (IDS vs. ADS), the multiple speakers and the uneven occurrence frequency of the tags. Notice however that the training and test speakers are the same in this database, which is also most relevant to CLA and SGML. It is remarkable to observe that the NMF-based recognition can successfully integrate correlated information streams. Given the small amount of errors, it is not possible to draw conclusions about the superiority of one configuration over another with any statistical significance, except that not using any co-occurrence (sound transition) statistics does not lead to good results (first row).

\section{PHONETIC ANALYSIS OF THE REPRESENTATIONS}

Section IV.B showed that the NMF method successfully learns keywords embedded in sentences and is able to recognize them later. The acoustic representations in the columns of $\mathbf{W}_{\text {aud }}$ are high dimensional and difficult to interpret, except that the corresponding columns in $\mathbf{W}_{g}$ show their relevance for activating each keyword. Typically, a column in $\mathbf{W}_{g}$ has only one (not multiple) large value or none at all, i.e. the acoustic representation contributes to only one keyword or to none. The latter case could be relevant for modeling the sound patterns of the carrier phrases (Table 2). In the former case, one could conclude that the model is learning words and not subword units, which also form a set of objects that can explain a scene as an additive combination, but which would require that multiple representations would contribute to the activation of a word.

\section{A. Analysis method}

To make the object models easier to interpret, a phonetic stream is added to the model. This stream is not used for the learning process, nor is it used to contribute to the recognition, but is merely added for analysis purposes. However, transcribing speech into phones automatically is error-prone. Instead of using just a single transcription, a phone lattice is generated with an automatic phone recognizer [14] using an acoustic model for Dutch trained on 50 hours of read speech

\begin{tabular}{|rrrrr|}
\hline$\tau_{1}$ & $\tau_{2}$ & $\tau_{3}$ & $N$ & $\begin{array}{r}\text { keyword error } \\
\text { rate }\end{array}$ \\
\hline 0 & - & - & 413 & $5.4 \%$ \\
\hline 2 & - & - & 38986 & $0.9 \%$ \\
\hline 5 & - & - & 48304 & $0.3 \%$ \\
\hline 10 & - & - & 50731 & $0.6 \%$ \\
\hline 2 & 5 & - & 87277 & $0.4 \%$ \\
\hline 2 & 10 & - & 89702 & $0.3 \%$ \\
\hline 5 & 10 & - & 99022 & $0.2 \%$ \\
\hline 2 & 5 & 10 & 137995 & $0.2 \%$ \\
\hline
\end{tabular}

Table 3: tag recognition results on the Dutch ACORNS [12] database. The leftmost 3 columns give the co-occurrence lag in frames (multiple of $10 \mathrm{~ms}$ ).

from the publicly available CGN corpus. For utterance $t$, the posterior probability $\gamma_{t, i}$ of the $i$-th arc in the lattice is computed according to [15] and the posterior probability $P_{t, k}$ of the $k$-th node is computed as well. The co-occurrence probability of phones $m$ and $n$ of adjacent arcs is accumulated over the lattice by constructing the sum:

$$
\left[\mathbf{C}_{t}\right]_{m n}=\sum_{\substack{\operatorname{arc} i \text { with } \\ \phi(i)=m}} \sum_{\substack{\operatorname{arc} j \text { with } \\ \phi(j)=n}} \delta_{\omega(i), \alpha(j)} \frac{\gamma_{t, i} \gamma_{t, j}}{P_{t, \omega(i)}}
$$

where $\alpha(i)$ and $\omega(i)$ are the start and end node of the $i$-th arc and $\phi(i)$ is its phone identity and $\delta_{k, l}$ is Kronecker's delta. Finally, the phone co-occurrence counts of all $T$ utterances are stacked in an observation matrix:

$$
\mathbf{V}_{P}=\left[\operatorname{vec}\left(\mathbf{C}_{1}\right) \quad \cdots \quad \operatorname{vec}\left(\mathbf{C}_{T}\right)\right]
$$

and the NMF description is augmented with the phonetic feature stream:

$$
\left[\begin{array}{c}
\mathbf{G} \\
\mathbf{V}_{\text {aud }} \\
\mathbf{V}_{P}
\end{array}\right] \approx\left[\begin{array}{c}
\mathbf{W}_{g} \\
\mathbf{W}_{\text {aud }} \\
\mathbf{W}_{P}
\end{array}\right] \mathbf{H}
$$

Subsequently, the NMF problem (6) is solved under the constraint that $\mathbf{W}_{g}, \mathbf{W}_{\text {aud }}$ and $\mathbf{H}$ are already known. With this constraint, we impose that the existing parts representation in the acoustic space is unchanged while the joint model also needs to explain the phonetic stream as well as possible. In other words, $\mathbf{W}_{P}$ hooks into the existing model and associates a phonetic co-occurrence model to each acoustic representation that was previously learned without phonetic information. Thanks to this phone co-occurrence model, it is now possible to measure the similarity of the representations to a reference phonetic transcription. To this end, the reference phone string is first transformed to a chain of arcs, where each arc receives unit posterior probability. Then the reference phone cooccurrence count vector $\mathbf{c}_{\text {ref }}=\operatorname{vec}\left(\mathbf{C}_{\text {ref }}\right)$ is formed as in (5) for this particular lattice and normalized to sum to unity. The divergence measure between the reference transcription and the $k$-th column of $\mathbf{W}_{P}$ (also normalized to sum to unity) is now: 


$$
D\left(\mathbf{c}_{\mathrm{ref}} \| \mathbf{W}_{: k}\right)=\sum_{i=1}^{N}\left(\left[\mathbf{c}_{\mathrm{ref}}\right]_{i} \log \frac{\left[\mathbf{c}_{\mathrm{ref}}\right]_{i}}{\mathbf{W}_{i k}}\right)
$$

A graphical representation of these divergences between $\mathbf{W}_{\text {aud }}$-columns for the model with $\tau_{1}=2, \tau_{2}=5$ and $\tau_{3}=10$ and the canonical transcription of each of the 37 words in the vocabulary is given in Figure 1. The columns have been permuted such that $\mathbf{W}_{g}$ has its largest elements along the main diagonal. The negative divergences (7) are exponenttiated and normalized to sum to one over all candidate models. The resulting normalized divergence is a number between 0 (poor match) and 1 (canonical transcription with best match) where high values (white) indicate that the part (W-column) responds most to the word.

\section{B. The keyword models}

The first 13 columns of $\mathbf{W}$ model the tags by construction. From Figure 1 it is seen that each tag model responds well to one word from Table 1. However, some carrier words also produce good matches on some keyword models. Acoustic similarity could be a reason for a good match, e.g. "auto" en "nou" are short words with a common vowel. However, this cannot be an argument for "komt" producing a good match on "mama" and "papa". Closer inspection reveals that the carrier "en daar komt $<$ key $>$ " is only used with these two keywords and that "komt" is not used in any other context. Hence, the word "komt" helps to recognize these 2 keywords. The association is not perfect, since "mama" and "papa" do occur in other carriers. Similarly, "dag", "hallo" and "hee" are only used with the proper names, which leads to an association effect. Finally, Dutch grammar imposes to use the definite articles "de" with "auto", "luier", "schoen" and "telefoon", while "het" is used with "bad", "boek" and "flesje". Also these associations are apparent from Figure 1.

\section{The carrier models}

First notice that unlike the tag models, the carrier models are not essential for recognition (but improve performance). Since NMF is an approximate decomposition, differences between data and model can be tolerated. If recognition is attempted using only the 13 first columns of $\mathbf{W}$, tag error rates of $13 \%$ to $20 \%$ are observed, depending on the values of $\tau$. Hence it is advantageous for recognition accuracy to not only rely on the tag models, but also try to explain the acoustic cooccurrences caused by the carrier sentences.

The relation between the 12 rightmost columns in Figure 1 and the carrier vocabulary is not that clear for multiple reasons. First, quite different factorizations can lead to local minima of the KLD that are nearly equal. This non-uniqueness could also be attributed to the fact that multiplicative updates for non-negative matrix factorization only guarantee nonincrease of the divergence and may exhibit problematic convergence behavior [16]. But even in ideal cases where the global minimum is attained, the number of contexts in which the objects (carrier words) occur must be large enough in order to be found as objects, or in terms of [9], the weights are not "sufficiently spread due to the database design. Secondly, the acoustic confusability of the words used in the carrier phrases blurs the divergence picture. Eight words contain only two phones, while pairs like "waar"/"daar" or "wat"/"dat" are acoustically similar. Third, the 12 columns (14 through 25 ) of W spent on the carrier phrases do not suffice to model 24 words individually. Fourth, some words always occur together, such as the separable verb "wijs aan" and one cannot expect to find individual models for the components. A lexical interpretation of the carrier columns is given in Table 4 .

\section{DISCUSSION}

The NMF-based method for acquiring speech differs in many respects from the HMM paradigm. NMF can infer its models from weak supervisory data: in the experiments, the carrier phrases were not transcribed orthographically as would be common with HMMs training. Moreover, the supervisory information could easily be a set of features derived from other modalities (see section II.A), which is not the case for HMMs. The resulting model also operates very differently from HMMs: it will compute word activations from observations acquired over some time period (and possibly from multiple modalities). At no point word segmentations are actually computed, which finds cognitive justification in the observation that human speech recognition is robust to crossword assimilation effects, which actually question the

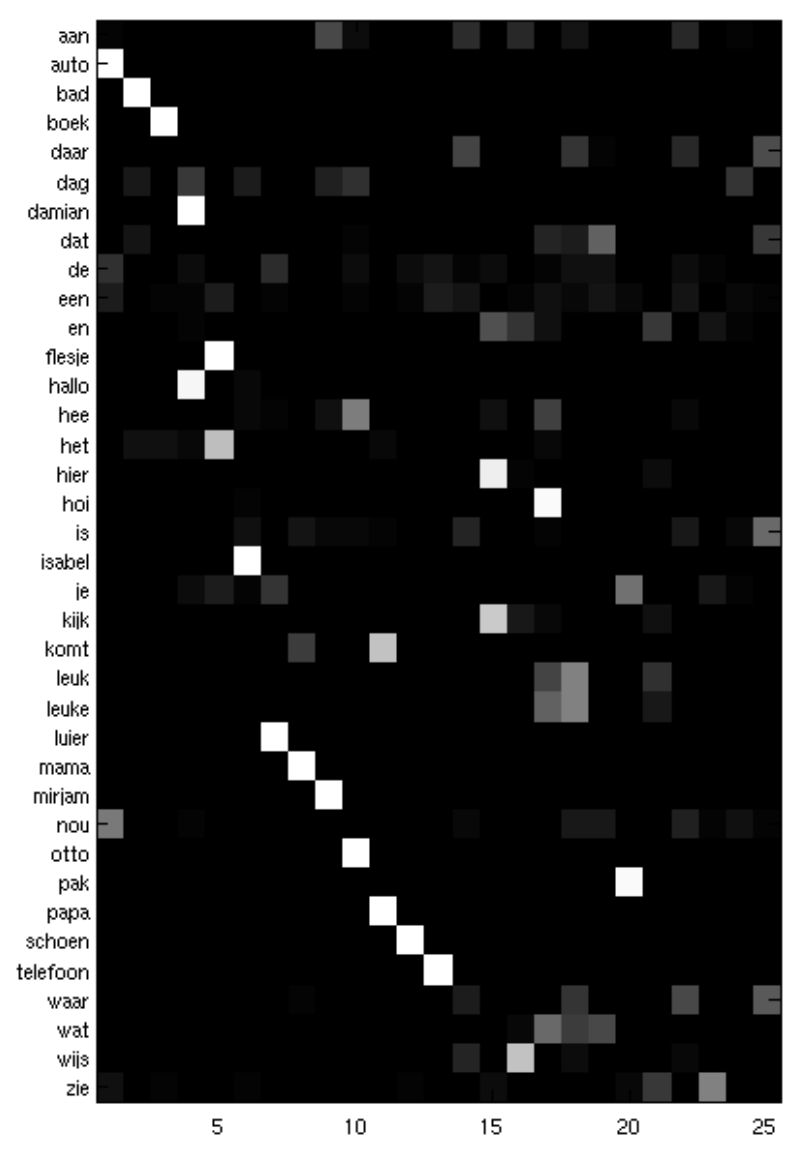

Figure 1: normalized divergence between canonical transcriptions (ordinate) and the columns of W (abscissa). White represents close match.

relevance or even existence of word boundaries. A downside 


\begin{tabular}{|cl|}
\hline Column & word / multi-word-expression \\
\hline 14 & "wijs ... aan" + "waar is" + "daar is" \\
\hline 15 & "kijk" + "en hier" \\
\hline 16 & "wijs ... aan" \\
\hline 17 & "hoi" + "hee" \\
\hline 18 & "wat ... leuk(e)" \\
\hline 19 & "wat/dat" \\
\hline 20 & "pak je" \\
\hline 21 & "zie" \\
\hline 22 & "waar" \\
\hline 23 & "zie je" \\
\hline 24 & "dag" \\
\hline 25 & "waar is" + "daar is" + "dat is" \\
\hline
\end{tabular}

Table 4: matching columns of $\mathbf{W}$ (left) with words and multi-word expressions in the carriers (right).

of not inferring word-level segmentations is that word activation does not reveal word order: only unordered word detection within a speech analysis window can be achieved. This can be cured by estimating word locations and with sliding window techniques [17].

A final note concerns with the cognitive plausibility of the NMF-based learning. The training and testing phases were separated explicitly in this paper, while in CLA and SGML, learning is incremental without a clear cut end of training. Therefore, the NMF learning has been made incremental in a Bayesian framework [18], so learning never ends while new experiences slowly supersede the older ones.

\section{CONCLUSION}

This paper first gave an account of the NMF-based approach to vocabulary acquisition form multimodal data. NMF is able to find descriptions of objects that recur in different configurations in a collection of scenes. A histogram of sound co-occurrence events is motivated by psycholinguistics and leads to good performance in the small vocabulary task attempted. A phonetic analysis shows that the learned representations model whole keywords rather than subword units. In our experiments, not enough representations were available to model all carrier words, leading to representations that fire on multiple carrier words. However, carrier word models, even if inaccurate, are indispensable for good keyword accuracy.

\section{ACKNOWLEDGMENT}

This research was funded in part by IWT-SBO project ALADIN contract 100049 and by the European Commission, under contract number FP6-034362 (ACORNS project).

\section{REFERENCES}

[1] C. Snow and C. Ferguson, "Talking to Children: language input and acquisition", Cambridge: Cambridge University Press, 1977

[2] A. L. Thomaz, and C. Breazeal, "Transparency and Socially Guided Machine Learning", In Proceedings of the 5th International Conference on Developmental Learning (ICDL), 2006.
[3] P. Nowell and R.K. Moore, "The application of dynamic programming techniques to non-word based topic spot-ting", in Proc. Eurospeech, pages 1355-1358, Madrid, Spain, 1995

[4] A. Park and J. Glass, "Towards unsupervised pattern discovery in speech," in Proc. ASRU, San Juan, Puerto Rico, 2005, pp. 53-58.

[5] S. Deligne and F. Bimbot, "Inference of variable-length linguistic and acoustic units by multigrams," Speech Communication, vol. 23, no. 3, pp. 223-241, 1997.

[6] D. K. Roy and A. K. Pentland, "Learning words from sights and sounds: a computational model", Cognitive Science 26 (2002), 113-146

[7] D. Lee, and H. Seung, "Algorithms for non-negative matrix factorization," Advances in Neural Information Processing Systems, vol. 13, pp. 556-562, 2001

[8] T. Nakamura, T. Nagai and N. Iwahashi, "Grounding of Word Meanings in Multimodal Concepts Using LDA," in Proc. IEEE/RSJ International Conference on Intelligent Robots and Systems, 2009

[9] H. Lauberg, "Uniqueness of Non-Negative Matrix Factorizatioin," in $14^{\text {th }}$ IEEE workshop on Statistical Signal Processing, pp. 44-48, 2007

[10] D. Lowe, "Object recognition from local scale-invariant features," in Proceedings of the International Conference on Computer Vision, pp. 1150-1157, 1999

[11] J. Saffran, R. Aslin, and E. Newport, "Statistical learning by 8-monthold infants," Science, vol. 274, no. 5294, pp. 1926-1928, 1996

[12] L. Boves, L. ten Bosch, and R. Moore, "ACORNS - towards computational modeling of communication and recognition skills", in Proc. IEEE conference on cognitive informatics, pages 349-356, 2007

[13] V. Stouten, K. Demuynck, K. and H. Van hamme, "Discovering Phone Patterns in Spoken Utterances by Non-negative Matrix Factorisation." IEEE Signal Processing Letters, volume 15, pages 131-134, 2008.

[14] K. Demuynck, T. Laureys, D. Van Compernolle and H. Van hamme, "FLaVoR: a Flexible Architecture for LVCSR.", in Proc. European Conference on Speech Communication and Technol-ogy, pp. 1973-1976, Geneva, Switzerland, September 2003.

[15] F. Wessel, R. Schluter, K. Macherey and H. Ney, "Confidence measures for large vocabulary continuous speechrecognition", IEEE Transactions on Speech and Audio Processing, Volume 9, Issue 3, pp. 288 - 298, 2001

[16] B. W. Berry, M. Browne, A. N. Langville, V. P. Pauca and R. J. Plemmons, "Algorithms and applications for approximate nonnegative matrix factorizations", Computational Statistics \& Data Analysis 52(1), pp. 155-173, 2007

[17] H. Van hamme, "HAC-models: a Novel Approach to Continuous Speech Recognition," In Proc. International Conference on Spoken Language Processing, pp. 2554-2557, 2008.

[18] J. Driesen and H. Van hamme, "Modelling Vocabulary Acquisition, Adaptation and Generalization in Infants using Adaptive Bayesian PLSA," Neurocomputing, in press. 